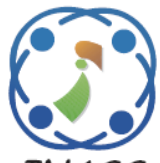

\title{
Enhancement of Wind Farm Design by Using Biogeography based Optimization
}

\author{
Mohammed Amine Hassoine ${ }^{1 *}$ \\ Fouad Lahlou ${ }^{1}$ \\ Adnane Addaim² \\ ${ }^{1}$ Faculty of Sciences, Ibn Tofail University, 14000 Kenitra, Morocco \\ ${ }^{2}$ Mohammadia School of Engineers, Mohamed V University, Rabat, Morocco \\ ${ }^{3}$ Advanced Systems Engineering Laboratory, National School of Applied Sciences, \\ Ibn Tofail University, 14000 Kenitra, Morocco \\ * Corresponding author's Email: mohamedamine.hassoine@uit.ac.ma
}

Abdessalam Ait Madi ${ }^{3}$

\begin{abstract}
This paper investigates the optimal layout of wind turbines (WTs) within a wind farm. Finding the best placement of WTs in a wind farm is a challenging process due to the existence of multiple wake effects. A biogeography based optimization (BBO) algorithm method is proposed to search for the optimal location of WTs in a wind farm (WF), to maximize the power produced by the WF and improve the annual economic performance of the WF. A wind turbine (WT) that operates in the wake of one or more other turbines is subject to lower flow and therefore produces less power. When designing a wind project, the arrangement of the turbines with each other in a wind farm is a very important factor. The best layout of a wind farm is to achieve the optimal placement of the turbines in relation to each other in a given area to maximize the efficiency of the whole wind farm and reduce its cost. A dense configuration would result in considerable power losses. Each turbine must have a sufficient distance from other turbines in the WF where the optimal number of turbines should be placed. The BBO approach is conducted on a 2 $\mathrm{km} \times 2 \mathrm{~km}$ wind farm assuming a constant wind speed of $12 \mathrm{~m} / \mathrm{s}$ with a fixed wind direction, for solving the wind farm layout optimization problem in two different configurations which include 26 and 30 WTs respectively. A comparison of the results obtained with the previous studies shows that the $\mathrm{BBO}$ is more efficient in terms of maximizing power output and economic profitability of the same wind farm model, which validates that BBO performs effectively in optimizing WTs placement within WF. BOO provides the greatest improvement in the optimal layout, for example, in the case of the layout for $30 \mathrm{WTs}$. The power output reaches $15,383 \mathrm{KW}$, the agreement between the ideal and the optimal layout is more favorable. The difference in output power is only $169 \mathrm{KW} \mathrm{(1 \% ).} \mathrm{Knowing} \mathrm{that} \mathrm{the} \mathrm{ideal} \mathrm{layout}$ is reached if all WTs receive a wind flow with a maximum wind speed of $12 \mathrm{~m} / \mathrm{s}$. Furthermore, a case study of wind turbine layout optimization using the BBO program on the Alta X wind farm has been performed under variable wind speed and variable wind direction. The results indicate that the optimized layout of the Alta X wind farm achieves a $12 \%$ increase in the power output for a similar cost when compared to the original layout of Alta X. It is also more appropriate for evaluating the wind farm layout in the Wind Power Project (WPP).
\end{abstract}

Keywords: Biogeography based optimization, Layout optimization, Wind farm, Wake model, Wind turbine.

\section{Introduction}

In recent years, the production of wind energy is in full expansion. Its advantages are numerous: first of all, it is a non-polluting renewable energy that contributes to a good air quality and to the fight against the greenhouse effect. The transition to renewable energies remains an essential issue for the world's economic future, as energy consumption is becoming enormous and continues to increase worldwide. Energy supply is mainly provided by fossil fuels, which remain the world's leading source: in 2019, coal, gas, and oil accounted for $84.3 \%$ of global energy consumption, compared to $11 \%$ for renewables and $4 \%$ for nuclear. [1] The share of renewable energy continues to grow every year, as reported by the International Energy Agency, the COVID-19 pandemic has not slowed the development of renewable energy [2]. While global electricity consumption decreased in 2020, renewable energy production increased up to $7 \%$. 
This year, $200 \mathrm{GWh}$ of new generation capacity was added, an increase of $4 \%$. Wind power capacity has increased by 60.4 gigawatts, or 19 percent, relative to 2018. This was one of the highest growth rates reported by the global wind industry, in 2019 global wind power capacity has reached $650.8 \mathrm{GW}$ [3].

The wind farm project (WFP) is especially complex to carry out, due to its multidisciplinary aspect. The identification of favorable sites for the implementation of a wind farm implies the availability of wind data for the location. The final selection of the site is based on a set of measurements to establish the wind data of the site. For that purpose, measurement surveys are conducted using measuring masts. They usually last 12 months. The main measurements are wind speed and direction. A wind farm operates best when winds are regular and frequent. Furthermore, the efficiency of a WT also depends on its placement with the other turbines. Separation of WTs and their position are crucial for the profitability of this kind of project. A wind turbine installed to take advantage of the prevailing wind is likely to disturb other neighboring turbines located downstream, due to the effects of blade movement. The wake effect is generated by the decrease in wind speed and the increase in turbulence, resulting not only in production losses for the downstream turbines but also in fatigue loads and premature wear of the installations. The design of a WF is generally described as a wind farm layout optimization problem (WFLOP). In the existing literature, various studies have investigated how to optimize the arrangement of wind turbines relative to each other to achieve maximum power output at a minimum cost.

Various heuristic optimization approaches have been used for solving the WFLOP. Mosetti et al. [4] and Grady et al. [5] solved the wind turbine positioning problem using a binary coded genetic algorithm (GA) to maximize energy production. Emami et al. [6] determined the optimal placement of wind turbines using GA with control on cost. Wan et al. [7] proposed a GA algorithm to find the optimal micrositing of WTs by incorporating more appropriate models of wind speed distributions and turbine power curves.

González et al. [8] employed GA to achieve a more efficient optimal solution for optimizing the WTs layout by applying a more realistic cost model taking into account the initial investment, production, operation, and maintenance costs of a wind farm. Mittal [9] proposed a GA to optimize the placement of WTs in a large WF to minimize the cost per unit of power.

Chen et al. [10] used the greedy algorithm to optimize the placement of WTs. Gao et al. [11] and
Hassoine et al. [12, 13] proposed a multi-population genetic algorithm for solving WF layout optimization problem. Using the same WF and cost models as Mosetti, Pookpunt [14] and Asaah et al. [15] demonstrated optimal placement using particle swarm optimization to maximize energy production. Bansal [16] and Pouraltaf et al. [17] introduced a biogeography based optimization to solve WFLOP.

This paper proposes an approach to optimize the layout of a wind farm using BBO. Firstly, this method has been evaluated and compared with the results of previous studies in the literature, with the aim of finding the optimal placement of multiple WTs. Secondly, the BBO approach has been used to optimize the layout of the Alta X wind farm. The simulation results indicate that the proposed method is capable to find the best layouts that surpass the current design by increasing power and efficiency in a reasonable area. It is found that the proposed method is more promising and has a greater improvement in terms of maximum energy production compared to other previously studied methods. The remainder of this paper is organized as follows. Section 2 presents wind farm models and problem definition. Section 3 describes the optimization process for the wind farm layout. The results of WF layout optimization and discussion are shown in Section 4. In Section 5, the layout optimization of Alta $\mathrm{X}$ is investigated and discussed. The conclusion of this proposed work is given in section 6 .

\section{Wind farm models and problem definition}

\subsection{Power calculation and wake model}

The energy received by the WT depends on the mass and speed of the wind. The kinetic energy of the wind produces a rotational motion of the turbine rotor. According to Betz's limit, the wind turbine can convert up to 16/27 (or 59\%) [18] of the kinetic energy of the wind into mechanical energy. The power output of the turbine can be expressed by Eq. (1):

$$
P_{w t}=\frac{1}{2} \eta \rho A u^{3}
$$

$P_{w t}$ is the power output, A is the area of the circle with a radius equal to the length of a blade, $u$ is the wind speed, $\eta$ is the efficiency of WT and $\rho$ is the density of the air.

In the present investigation, the wind farm is assumed to be a square area of $2000 \mathrm{~m} \times 2000 \mathrm{~m}$. The farm is divided into 100 unit areas (200 m x $200 \mathrm{~m})$. 


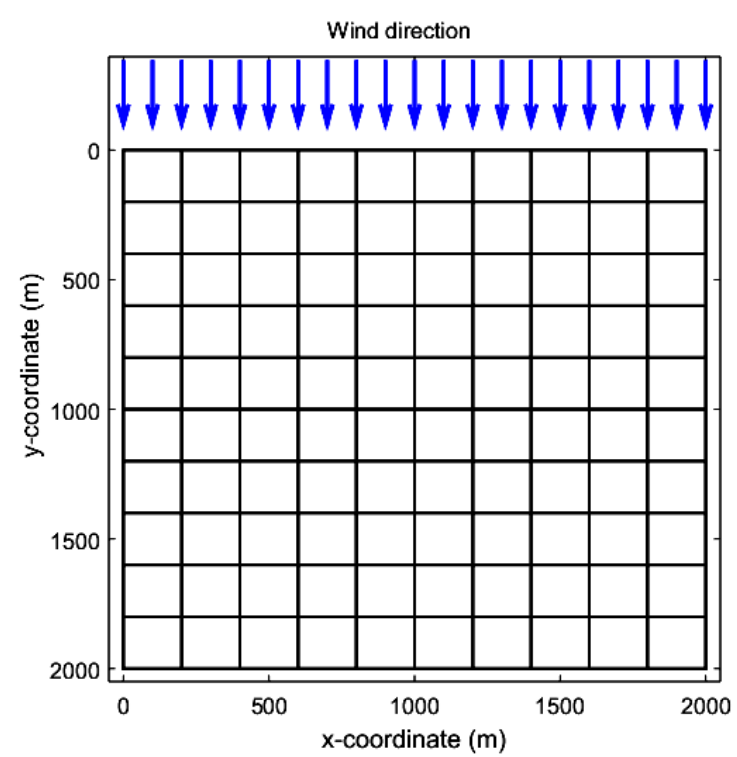

Figure. 1 Model of the wind farm

Table 1. Wind turbine parameters

\begin{tabular}{|c|c|}
\hline Parameters & Specifications \\
\hline Rated power $(\mathrm{kW})$ & 630 \\
\hline Cut-in wind speed (m/s) & 2.3 \\
\hline Rated wind speed (m/s) & 12.8 \\
\hline Cut-out wind speed (m/s) & 18 \\
\hline Hub height $(\mathrm{m})$ & 60 \\
\hline Rotor diameter(m) & 40 \\
\hline
\end{tabular}

A unidirectional wind speed is assumed of $12 \mathrm{~m} / \mathrm{s}$ coming from the north as seen in Fig. 1.

The WT chosen for the present study is the same as that selected in previous studies $[4,5]$. Table 1 shows the technical characteristics of the WT. The thrust coefficient is assumed to be constant, for a speed of $12 \mathrm{~m} / \mathrm{s}$, with a value of 0.88 [4]. The surface roughness of the WF is 0.3 .

The efficiency is considered equal to $40 \%$ [4], the Eq. (1) will be used as given by the Eq. (2) shown below:

$$
\begin{gathered}
P_{w t}=\frac{40}{100} \times \frac{1}{2} \times 1.2 \times \pi \times(20)^{2} \times u^{3} \\
P_{w t}=0.3 u^{3} \mathrm{KW}
\end{gathered}
$$

The power of WT, is explained by the Eq. (3) as follows:

$$
P_{w t}(\mathrm{u})=\left\{\begin{array}{c}
0 ; u<2.3 \\
0.3 u^{3} ; 2.3 \leq u \leq 12.8 \\
630 ; 12.8 \leq u \leq 18 \\
0 ; u \geq 18
\end{array}\right.
$$

where the wind speed $\mathrm{u}$ is given in $\mathrm{m} / \mathrm{s}$ and the power output $P_{w t}$ in $\mathrm{kW}$.

When a WF is composed of multiple wind turbines, the wind farm power output is determined by the Eq. (4):

$$
P_{w f}=\sum_{i=1}^{N} P_{i}
$$

where $P_{w f}$ is power output of WF, N is the number of turbines and $P_{i}$ is the power of the i-th WT.

The efficiency of the WF is defined as the ratio of the power of the WF to the summation of the power output of the individual WT without wake effects. This efficiency is given as shown in Eq. (5):

$$
\eta_{w f}=\frac{P_{w f}}{\sum_{i=1}^{N} P_{s i}}
$$

Where $\eta_{w f}$ efficiency of WF, $P_{s i}$ is the power of the $\mathrm{i}$-th wind turbine if it works as a separate WT.

When the wind passes across the rotor of a wind turbine, a wake is generated behind it, the wake extends with distance. The speed of the turbines positioned downstream of the other turbines has a speed deficit. To estimate this deficit, we used the Jensen model [19]. This model assumes that the wake grows linearly with distance. The calculation of the wind speed deficit at the downstream distance is given by Eq. (6):

$$
\frac{u_{w}(x)}{u_{0}}=1-\frac{2 a}{\left(1+2 \alpha\left(\frac{x}{D_{r}}\right)\right)^{2}}
$$

where $u_{0}$ is the mean wind speed, $x$ is the downstream distance, $a$ is the axial induction factor, $u_{w}$ wind speed of wake at downstream distance, $\alpha$ is the decay factor and $D r$ is the downstream rotor diameter of the turbine. The decay factor $\alpha$ describes how the wake expands. The Wake width is defined as shown in Eq. (7):

$$
D_{w}(x)=D_{r}+2 \alpha x
$$

where $D_{w}$ is the diameter of the wake at downstream distance.

The wake decay constant (decay factor) depends on the hub height $(Z)$ and the surface roughness height $\left(\mathrm{Z}_{0}\right)$. This decay factor can be calculated from Eq. (8) [18]: 


$$
\alpha=\frac{0.5}{\ln \left(z / z_{0}\right)}
$$

The downstream rotor diameter (Dr) of the turbine is related to the wind turbine rotor diameter (D) through the Betz relations as given in Eq. (9) [18]:

$$
D_{r}=D \sqrt{\frac{(1-a)}{(1-2 a)}} ; a=\frac{1-\sqrt{1-C_{T}}}{2}
$$

Here, $C_{T}$ is the thrust coefficient of the turbine and $\mathrm{a}$ is the axial induction factor of WT.

When a wind turbine is exposed to the wake of multiple turbines, various methods can be employed to combine the multiple wakes. Among those are the sum of squares of velocity deficits, the energy balance method, the geometric sum, and the linear superposition [20, 21]. In the present article, we use the sum-of-squares model. The equation for the sum of squares of velocity deficits is given by Eq. (10) as follows [22].

$$
\left(1-\frac{u_{i}}{u_{0}}\right)^{2}=\sum_{\substack{j=1 \\ j \neq i}}^{N}\left(1-\frac{u_{i j}}{u_{0}}\right)^{2}
$$

where $u_{i}$ velocity of wind turbine $i, u_{i j}$ is the velocity in the downstream wake region of the turbine $\mathrm{j}$ which affects turbine $i$ and $u_{o}$ is the mean wind speed.

\subsection{Cost model and objective function}

The predicted cost of a WF per year was determined by Mosetti et al. [4]. using the number of WTs N. The annual non-dimensional cost of one WT is assumed to be 1 . For an additional turbine, the maximum possible cost reduction is equal to $1 / 3$. The cost function formula is given by Eq. (11).

$$
\text { Cost }=N\left(\frac{2}{3}+\frac{1}{3} e^{-0.00174 N^{2}}\right)
$$

where Cost is the cost per year of a wind farm.

The first derivative of the cost function cost grows linearly at $\mathrm{N}>30$. This means that at $\mathrm{N}>30$ the cost of the $(1+\mathrm{N})$ th turbine is higher than the cost of the $\mathrm{N}$ th turbine. When looking for the best wind farm layout that minimizes the unit cost of energy produced. The goal can be formulated as maximizing the power produced by the wind farm while minimizing the installation cost of the wind farm. An objective function will be used to minimize the cost per unit of energy produced, which is the effective cost per KW (Kilo Watt) of energy produced. This objective function is expressed by the following Eq. (12).

$$
\text { Objective }=\frac{\operatorname{Cos} t}{p_{w f}}
$$

where $P_{w f}$ is the power output of the wind farm.

\subsection{Wind farm layout optimization problem definition}

Assuming the WF has $\mathrm{N}$ wind turbines and, the width of the WF is $2000 \mathrm{~m}$. The wind farm layout optimization problem is to determine the best placement of the WTs in the wind farm area, such that the output power is maximized while cost is minimized. Therefore, the aim is to know the $\mathrm{x}$ coordinates and $\mathrm{y}$-coordinates of $\mathrm{N}$ wind turbines when the objective function reaches the minimal value. All the WTs in the wind farm are supposed to be identical and have a similar power curve. The number of variables for optimization is $2 \mathrm{~N}(\mathrm{x} ; \mathrm{y})$, the range of each variable is $[0,2000]$.

\section{Wind farm layout optimization process}

Wind farm layout optimization refers to the identification of optimal turbine positions in a specified area, that minimize the wake impacts with the condition of minimizing the cost and maximizing the power output of WF. In the present study, we propose the use of Biogeography-Based Optimization (BBO) for the design search of a wind farm. In this section, a description of the $\mathrm{BBO}$ process is presented.

The BBO method was proposed by Simon in 2008 [23] and was motivated by biogeography on the migration of species between habitats. The BBO method optimizes a given problem by iteratively and randomly improving the candidate solutions against an objective function (fitness).

The optimization process starts with the production of a finite number of selected individuals (habitats) by random selection in the search space, forming the initial population. Following evaluation of the initial population, certain individuals are selected to participate in the migration process that generates a new set of individuals. This phase is stochastic and depends on the emigration and immigration rates of the individuals concerned. The descendants will in turn be mutated. The mutation rate defines the proportion of the population that will be renewed at each generation. Elitism makes it 
Table 2. The parameters of BBO

\begin{tabular}{|c|c|}
\hline Parameters & Value \\
\hline Population size & 10 \\
\hline Mutation probability & 0.015 \\
\hline Elitism rate & 2 \\
\hline Iteration number & 10000 \\
\hline
\end{tabular}

possible to keep the best individuals found, as long as they are not surpassed by others. Finally, a replacement phase consists of substituting the parents for the new descendants to create a new population of the same size as at the beginning of the iteration.

Simon, in 2008 [23], has demonstrated that the BBO has good convergence characteristics for various benchmark functions when compared to other metaheuristics such as particle swarm optimization (PSO), ant colony optimization (ACO), and genetic algorithm (GA). Considering that the WF layout optimization problem has been solved for many years using PSO, ACO, and GA approaches. We can therefore summarize that the BBO presents a good alternative for WFLOP solution.

The WF receives a uniform and unidirectional wind speed. The calculation of the WF's power output requires the calculation of the speed of each turbine. The performance of the WF is evaluated by the fitness value using Eq. (12).

In a WF, the positions of the number of $(\mathrm{N})$ wind turbines are determined by two dimensional coordinates $(\mathrm{x} ; \mathrm{Y})$. The wind speed of each wind turbine is computed by using the Jensen wake model as showed in Eq. (6), and the superposition effects of multiple wakes upstream of each wind turbine are calculated employing the sum-of-squares method, as shown in Eq. (10). The power output of the individual turbines and, the power output of the WF are calculated by utilizing Eqs. (3) and (4).

The optimal layout is found through an iterative process by writing code in MATLAB based on BBO. The outputs of the computational results are the coordinates of the turbines, the wind speed of the individual turbines, the power output, and the levelized cost (fitness) of the WF. The process stops once the best fitness keeps the identical value for 500 generations. The BBO parameters are given in Table 2.

\section{Wind farm layout optimization results and discussion}

We consider a $2000 \mathrm{~m} \times 2000 \mathrm{~m}$ WF that is segmented in 100 squares $(200 \mathrm{~m} \times 200 \mathrm{~m})$. In contrast
Table 3. Comparison of Layouts

\begin{tabular}{|c|c|c|}
\hline & Layout1 & Layout2 \\
\hline Number of turbines & 26 & 30 \\
\hline Fitness value & 0.0014928 & 0.0014358 \\
\hline Power output (KW) & 13401.42 & 15383.85 \\
\hline Efficiency (\%) & 99.42 & 98.91 \\
\hline Number of iterations & 10000 & 10000 \\
\hline
\end{tabular}

to the earlier approaches in which a turbine would be positioned in the center of the square, the present work limits the minimum distance between any two adjacent turbines to $200 \mathrm{~m}$ (5D), and the turbines can be placed anywhere in the WF. To perform our proposed approach, we have developed a MATLAB code that uses the BBO algorithm. The output computational results are wind speed, total power output, and cost. The program will be terminated under the condition that the best fit will remain the same with no change in 500 iterations.

The BBO is implemented using the Jensen model for the computation of the wake effects. A population of ten individuals has been implemented to evolve over 10000 iterations. After the BBO program is run on two areas of investigation, two optimal layouts of the wind farm are achieved.

For the first area (layout 1), after 1139 iterations, the value of the best fit keeps nearly constant and the best layout of $26 \mathrm{WTs}$ is attained for the best fitness value of 0.0014928 and the power output is 13401.42 $\mathrm{kW}$ with an efficiency of $99.42 \%$. Figs. 2 and 3, respectively, show the convergence plot of the fitness curve and the evolution of the power output for layout 1 .

In the second area (layout 2), the best layout for 30 WTs is achieved after 1612 iterations with the best fitness value of 0.0014358 and the power output is $15383.85 \mathrm{~kW}$ with an efficiency of $98.91 \%$. Figs. 4 and 5 respectively show the convergence plot of the fitness curve and the evolution of the output power for layout 2. Table 3 shows a comparison of the two optimal layouts.

The optimized result for 30 WTs shows, on the one hand, that the optimal layout for $30 \mathrm{WT}$ gives a lower cost than $26 \mathrm{WT}$. On the other hand, the layout for $26 \mathrm{WTs}$ gave a better performance, but for 4 more WTs, the search space became narrower to search for optimal placement, Fig. 7. shows a denser layout than in Fig. 6.

The results achieved using biogeography based optimization (BBO) are compared to the previous studies that are performed with various approaches. 


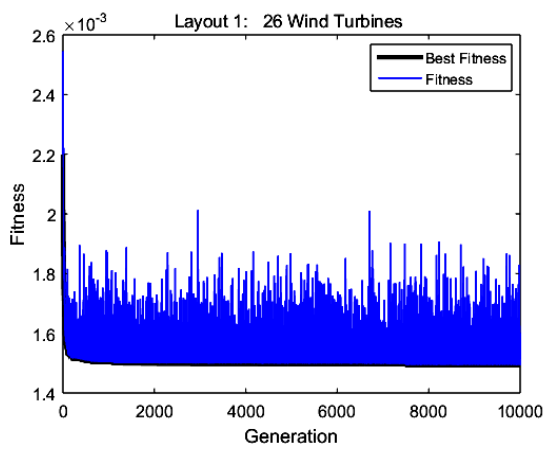

Figure. 2 Convergence curve of fitness for layout 1

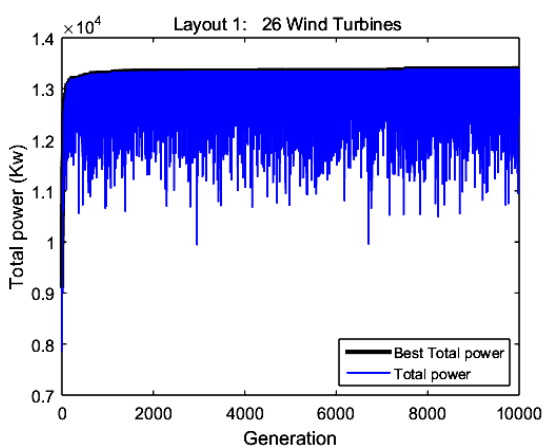

Figure. 3 Evolution of power output for layout 1

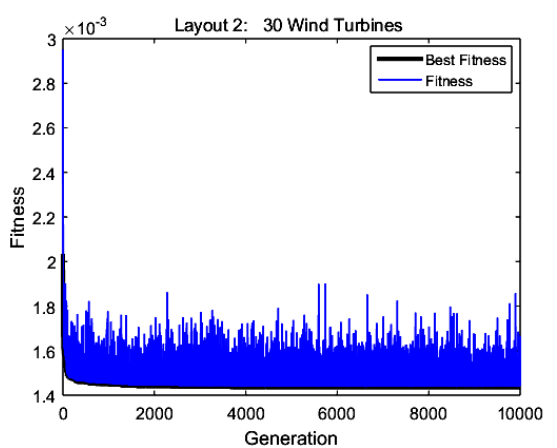

Figure. 4 Convergence curve of fitness for layout 2

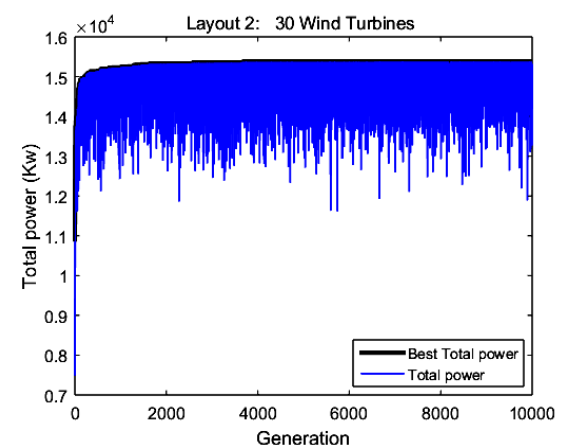

Figure. 5 Evolution of power output for layout 2

Like genetic algorithm (GA) and its variants such as real coded genetic algorithm (RCGA), multipopulation genetic algorithm (MPGA), real coded multi-population genetic algorithm (RCMPGA) and

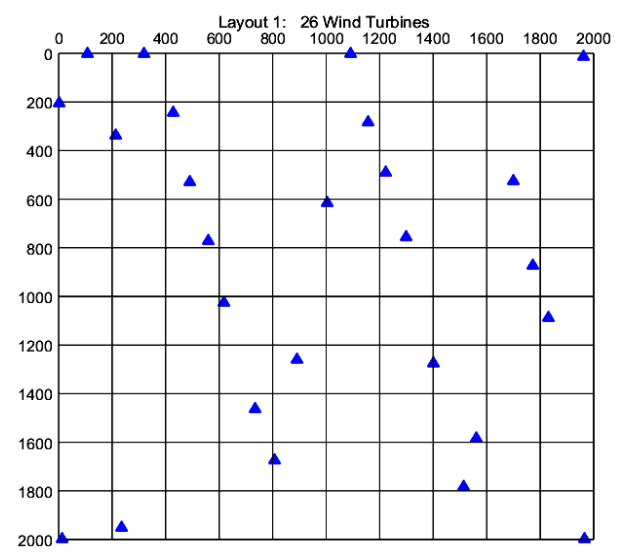

Figure. 6 Optimal layout of 26 WTs

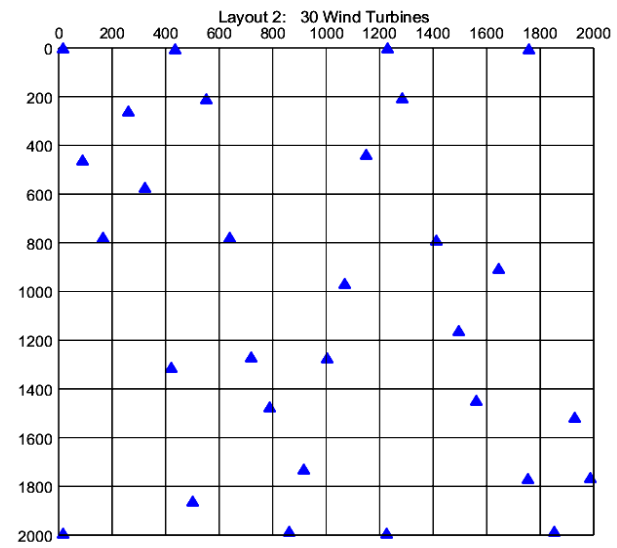

Figure. 7 Optimal layout of 30 WTs

enhanced genetic algorithm (EGA) as well as particle swarm optimization (PSO) and greedy algorithm (GRA).

Some comparisons of the fitness value and power output, as well as the efficiency of the results achieved by BBO and the previous articles, are reported in Table 4. Before discussing and comparing the results of Table 4. A brief description of the GA, PSO, and GRA approaches is presented below.

Genetic algorithms are search techniques inspired by the natural phenomena of genetics and evolution. GA is used to find optimal solutions to optimization problems. At the start of the optimization process, a set of solutions named individuals is randomly constructed, the solutions are coded by a binary or real coding. When solving the WFLOP, each solution is a possible layout of the turbines in the wind farm, the arrangement gives a different set of the $\mathrm{x}$ coordinates and $\mathrm{y}$-coordinates of $\mathrm{N}$ wind turbines. The individuals constitute one or more populations. Genetic reproduction is performed using crossover and mutation operators that recombine the best individuals. The evaluation of the population 
(solutions) is done by using the objective function (fitness). The individual selection is executed following a procedure that selects the parents according to a probability proportional to their relative value (fitness) and explores the solution area in search of better and better solutions. The optimization process ends if the best fitness is maintained without any change for a significant number of generations. Genetic algorithms perform well for complicated problems, however, take a long time to find an optimal solution.

In contrast to GA, the PSO approach does not have crossover and mutation operators. In PSO, possible solutions are named particles. A particle is an individual in a population or swarm. Each particle is characterized by a position and speed which include the value of the objective function. The position of a particle is the candidate solution. A particle searches for the optimal position in the search area of the wind farm by changing its velocity. The process is started when a population of solutions is constructed randomly, the search for optimal solutions is done by updating the generations. In PSO, the particles fly along the space of the problem following the current optimal particles. At each generation, the particles are updated according to the two "best" values. The first is the best possible solution (fitness) it has obtained so far. This value is denoted pbest. An additional value is the highest value achieved so far by any particle. This best value is a global value and named gbest. The best value of particle is the best location. Once the two best values are found, the particle updates its velocity and position based on the pbest and gbest values, so that the particles can gradually converge to a global minimum. Particle swarm optimization can converge faster than genetic algorithms, however, it can converge prematurely and be stuck in a local minimum, especially for complex problems.

A greedy algorithm is an algorithm that works step by step, the algorithm looks for a local optimal solution hoping to find a global optimal solution. The process includes two phases. The first phase begins with an empty layout. The turbines are inserted into the wind farm one by one by evaluating the objective function of a single turbine, a turbine added to the WF for the maximum wind speed. Each empty area is assessed. A new turbine is placed at the area of maximum evaluation value, the order of adding the turbines to the wind farm is stored. If the required number of turbines is reached, the phase is completed. In the second phase, a turbine is removed according to the order of the turbines in the addition process. To ensure that an optimal overall solution is obtained. Then a new optimal placement is found for the turbine with all empty areas. The turbine is replaced at the location where the maximum evaluation value is achieved. The process is terminated when all turbines pass through the relocation procedure. A greedy algorithm is easier to implement. However, the optimal global solution is not always reached.

The results of all studies in Table 4 were completed for identical simulation conditions. To solve the wind farm layout optimization problem, a wind farm $(2 \mathrm{~km} \times 2 \mathrm{~km})$ was employed, which was partitioned into 100 cells to place 26 and $30 \mathrm{WTs}$ respectively, the minimum spacing between two neighboring wind turbines is $200 \mathrm{~m}$, under conditions of uniform wind direction with a wind speed of $12 \mathrm{~m}$ /s.

The results in Table 4 show that multiple studies provided similar results, particularly for the $30 \mathrm{WT}$ case. Grady et al., Emami et al., and González et al. have found a power output of $14,310 \mathrm{KW}$ for an optimal configuration of 30 WTs. This case illustrates that the use of various population size and number of generations used in the genetic algorithms lead to the same outputs. For illustrative purposes. Grady et al. utilized a population of 600 individuals in 20 subpopulations to evolve over 3000 iterations. Gonzalez et al. used 100 individuals to evolve over 81 generations. In contrast, Emami et al. used 20 individuals to evolve over 150 generations.

In addition, the other approaches yielded similar values. In particular, Pookpunt employed particle swarm optimization, and Chen et al. used the greedy algorithm to find the optimal layout for $30 \mathrm{WT}$. It is important to note that the power output does not exceed $14,310 \mathrm{~kW}$. This limitation is caused by the fact that the wind farm is divided into 100 cells and the turbines are placed in the center of the cells, the width of each cell is equal to the five rotor diameters (5D).

In contrast to this restriction, the optimal layout of 30 WTs was improved by placing the turbines freely in the WF with a minimum distance of $200 \mathrm{~m}$ between two adjacent WTs.

Studies that adopted this strategy provided better results. However, the results were not similar. As a result, Mittal's work slightly increased the power output by $14,336 \mathrm{KW}$, Yin improved the layout of 30 WT, the power reaches $15,091 \mathrm{KW}$. The results of Wan et al. and Gao et al. indicate a significant improvement in the turbine layout, the power output reaches $15,220 \mathrm{KW}$ and $15,346 \mathrm{KW}$, respectively. Furthermore, the results of Hassoine et al. show a powerful improvement in the layout of $30 \mathrm{WTs}$. The output power reaches $15,362 \mathrm{KW}$. The proposed $\mathrm{BOO}$ provides the highest enhancement of the layout 30 WTs. The power output reaches $15,383 \mathrm{KW}$. 
Table 4. comparison of our present study and previous results

\begin{tabular}{|c|c|c|c|c|}
\hline Studies & Number of WT & $\begin{array}{c}\text { Fitness value } \\
\left(\times \mathbf{1 0} \mathbf{0}^{-3}\right.\end{array}$ & Power output(kW) & Efficiency (\%) \\
\hline Mosetti et al. [4] & 26 & 1.6200 & 12,352 & 91.65 \\
\hline Grady et al. [5] & 30 & 1.5440 & 14,310 & 92.02 \\
\hline Wan et al. [7] & 30 & 1.4513 & 15,220 & 97.87 \\
\hline Emami et al. [6] & 30 & 0.1221 & 14,310 & 92 \\
\hline González et al. [8] & 30 & 1.5440 & 14,310 & 92.02 \\
\hline Mittal [9] & 30 & 1.5410 & 14,336 & 92.18 \\
\hline Chen et al. [10] & 30 & 1.5440 & 14,311 & 92.02 \\
\hline Pookpunt [14] & 30 & 1.5440 & 14,310 & 97.50 \\
\hline Gao et al. [11] & 26 & 1.5230 & 13,141 & 98.67 \\
\hline Gao et al. [11] & 30 & 1.4400 & 15,346 & - \\
\hline Yin [24] & 30 & 1.4637 & 15,091 & 99.25 \\
\hline Hassoine et al. [12] & 26 & 1.4955 & 13,377 & 98.77 \\
\hline Hassoine et al. [12] & 30 & 1.4379 & 15,362 & 99.42 \\
\hline Proposed BBO & 26 & 1.4928 & 13,401 & 98.91 \\
\hline Proposed BOO & 30 & 1.4358 & 15,383 & 92 \\
\hline
\end{tabular}

For the proposed $\mathrm{BOO}$ results of $30 \mathrm{WTs}$, the agreement between the ideal and optimal layout is more favorable. Knowing that the ideal layout is achieved, if all WTs receive a wind flow with a maximum wind speed of $12 \mathrm{~m} / \mathrm{s}$, then the output power reaches $15,552 \mathrm{KW}$. Therefore, the difference in output power is only $169 \mathrm{KW}(1 \%)$.

In the case of 26 WTs. The output power reaches $13,478 \mathrm{KW}$ of the ideal layout. The proposed BOO provides the best results with the greatest improvement, the output power reaches $13,401 \mathrm{KW}$. Consequently, the difference of output power is only $77.4 \mathrm{KW}(0.57 \%)$. The results give a lower value of the objective function (fitness). This means a lower cost per unit of energy produced. The work of Mosetti et al. provides the lowest power output value of $12,352 \mathrm{KW}$. Mosetti et al. were the first to use genetic algorithms to solve WFLOP problems.

The proposed $\mathrm{BBO}$ simulation provides better results compared to the previous approaches. The results obtained indicate that the proposed method is more suitable for solving WFLOP. This performance is due to the fact that even though BBO is based on solution populations, the solutions are conserved and modified by the migration operator after each iteration considering the biogeographic roots of the algorithm. On the contrary, in other conventional approaches, such as genetic algorithms or particle swarms, new generations are formed at each iteration.

\section{Layout optimization of Alta $X$}

The comparative study started in Section 4, demonstrated the validity of the BBO approach, in the rest of this study, we apply the method under real wind conditions on an existing wind farm named Alta $\mathrm{X}$.

\subsection{Alta $X$ wind farm models and data source description}

The Alta X wind farm is part of the larger Alta Wind Energy Center (AWEC), which is located in southeastern Kern County in California, approximately 4.8 kilometers northwest of the city of Mojave, and $18 \mathrm{~km}$ east of the city of Tehachapi, Fig. 8 shows a map of Alta X wind farm (AXWF). AWEC is the third largest wind farm in the world [25] with an installed capacity of $1,548 \mathrm{MW}$. The AXWF is composed of 48 WTs of $2.85 \mathrm{MW}$ (GE 2.85-103) manufactured by General Electric. The coordinates of AXWF are $35^{\circ} 5^{\prime} 2.472^{\prime \prime} \mathrm{N}$ and $118^{\circ} 15^{\prime} 17.1^{\prime \prime} \mathrm{E}$. The numbers from 1 to 48 are used in the numbering scheme as seen in Fig. 9.

Alta $\mathrm{X}$ is located within the high desert plains and hills on the western edge of the Mojave Desert. The surface roughness value of 0.3 . The thrust coefficient is assumed to be constant for a speed of 10 to $11 \mathrm{~m} /$ 


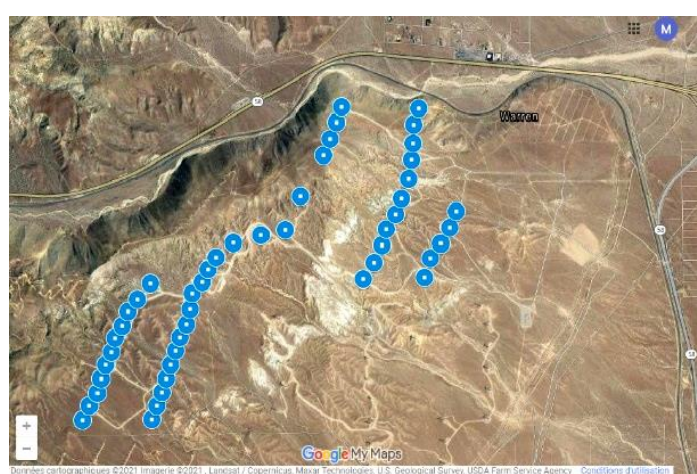

Figure. 8 Map of Alta X

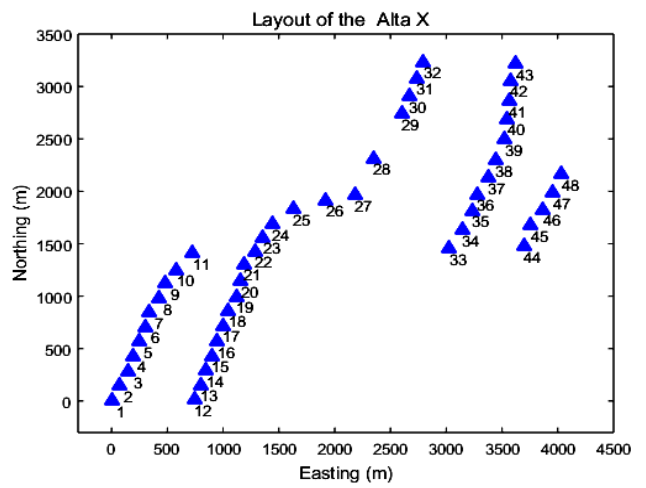

Figure. 9 Numbering scheme of Alta X

Table 5. Characteristics of WT.

\begin{tabular}{|c|c|}
\hline Parameters & Specifications \\
\hline Wind turbine model & GE 2.85-103 \\
\hline Rated power $(\mathrm{kW})$ & 2850 \\
\hline Cut-in wind speed (m/s) & 3 \\
\hline Rated wind speed $(\mathrm{m} / \mathrm{s})$ & 12.5 \\
\hline Cut-out wind speed $(\mathrm{m} / \mathrm{s})$ & 25 \\
\hline Hub height $(\mathrm{m})$ & 85 \\
\hline Rotor diameter $(\mathrm{m})$ & 103 \\
\hline
\end{tabular}

$\mathrm{s}$, with a value of 0.5 [26]. Table 5 shows the technical characteristics of the General Electric turbine (GE 2.85 -103) [27].

The frequency distribution of the wind speed has been described using the Weibull distribution function. Here a graphical method [28] was used in which the Weibull parameters were calculated using the least squares linear regression method [29]. The wind speed is extrapolated to the hub height of the WT using the power law. Here, we use one year (2012) of hourly wind data extracted from the NREL Wind Integration National Dataset [30], also known as the WIND Toolkit. Fig. 10 shows the hourly wind speed of AXWF at 80 meters, with an average of $10.43 \mathrm{~m} / \mathrm{s}$ marked by the green line. The values of the

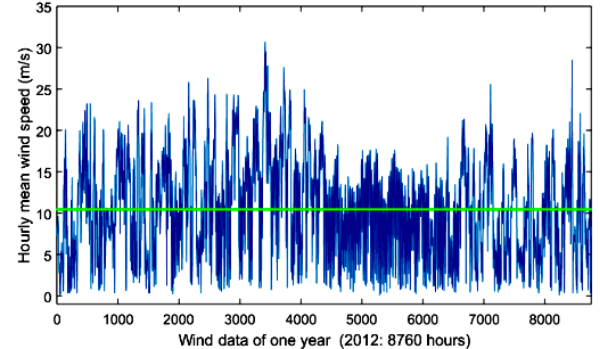

Figure. 10 Wind speed in Alta X, from 01 January 2012 to 31 December 2012

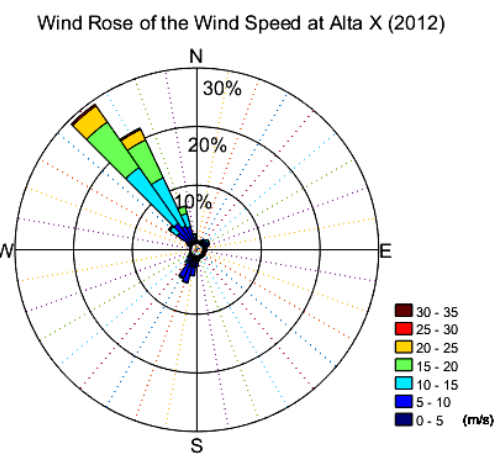

Figure. 11 Wind rose of wind speed of AXWF

shape parameter $(\mathrm{k})$ and the scale parameter $(\mathrm{c})$ are, respectively, 1.7227 and $11.7082 \mathrm{~m} / \mathrm{s}$. To give an overview of how wind speed and direction are distributed, the wind rose is used to illustrate the predominant wind directions at the Alta $X$ site. The predominant wind direction in this area is calculated over a period of 8670 hours, which shows that the wind direction is mainly generated from the WNW (West Northwest) and SW (Southwest) as seen in Fig. 11.

The mean yearly production of the Alta X between 2014 and 2019 is 347,161 MW [31]. The maximum production is achieved in 2018 when reaching 373,532 MW.

\subsection{Alta X layout optimization}

In the Alta X Layout Optimization process, wind speed data from NREL (2012) are considered. A population of fifteen individuals was allowed to evolve over 2000 iterations. After executing the BBO program for 1264 iterations, the results of the 48 WTs layout optimization in AXWF are achieved with the fitness value of 0.0445560 and the power output of $43683.18 \mathrm{~kW}$ with an efficiency of $31.93 \%$. Fig. 12 shows the optimal layout of Alta X. We can observe that the minimum distance constraint between two adjacent turbines is sufficiently respected. The placements achieved have a good distribution when 
Table 6. Comparison of results.

\begin{tabular}{|c|c|c|}
\hline & $\begin{array}{c}\text { Optimized } \\
\text { Layout }\end{array}$ & $\begin{array}{c}\text { Initial } \\
\text { Layout }\end{array}$ \\
\hline Number of turbines & 48 & 48 \\
\hline Power output (KW) & 43683.18 & 38973 \\
\hline Efficiency (\%) & 31.93 & 28.48 \\
\hline Energy (MWh) & 382664.65 & 341403.48 \\
\hline
\end{tabular}

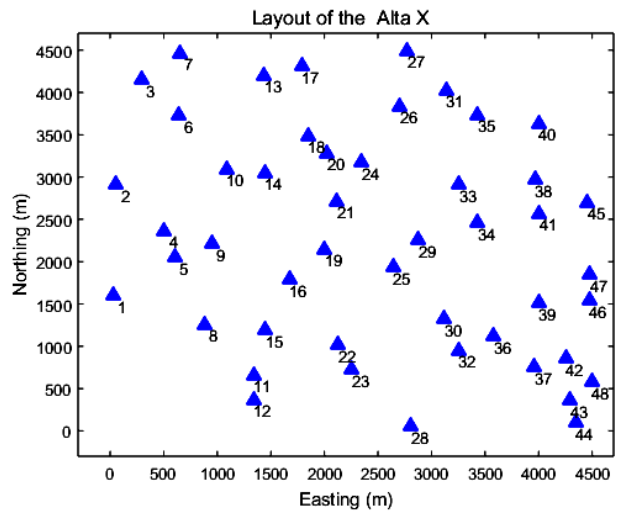

Figure. 12 Optimal layout of Alta X

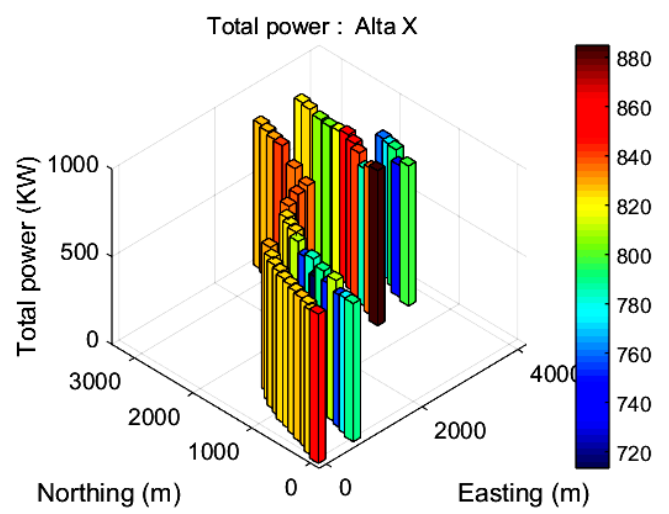

Figure. 13 WTs Power output of Initial Layout

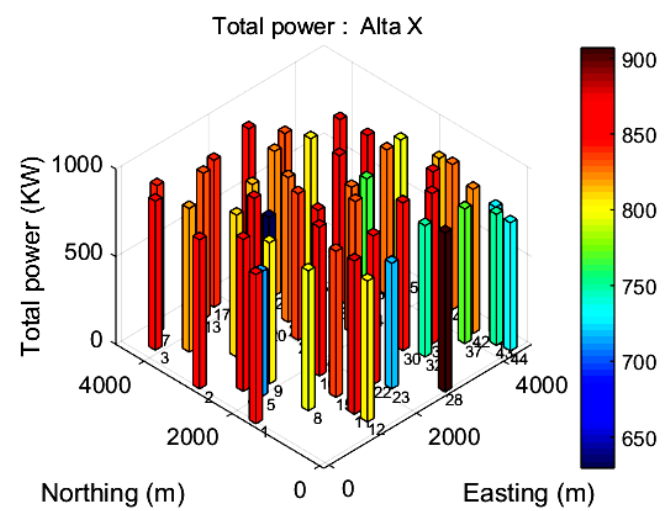

Figure. 14 WTs Power output of the optimized Layout

compared with the original layout of Alta $X$. The results are compared in Table 6 .

The power output of the wind farm is significantly improved (12\%). We can see in Fig. 14 that all turbines deliver the maximum power from the available wind conditions when compared with the power output of turbines within the initial layout in Fig. 13.

\section{Conclusions}

In this work, the optimal placement of turbines in a wind farm was solved using a biogeography based optimization approach and Jensen's wake model. Biogeography based optimization was applied to obtain the best placements of WTs, to maximize the power generated by the WTs, and improve the economic performance of the wind farm.

The $\mathrm{BBO}$ algorithm is applied to a $2 \mathrm{~km} * 2 \mathrm{~km}$ wind farm to solve the layout optimization problem in two different configurations. The outputs show that in the first configuration, the output power reaches $15,383 \mathrm{KW}$ for an optimal layout of $30 \mathrm{WTs}$, the agreement between the ideal layout and the optimal configuration is more favorable, the difference in output power is only $1 \%$. In the second configuration, the output power reaches $13,401 \mathrm{KW}$ for an optimal layout of $26 \mathrm{WTs}$, the difference in output power is only $0.57 \%$. The concordance between the ideal layout and the optimal configuration is higher. A case study of the Alta X wind farm was also performed with a variable wind speed and direction. The results obtained demonstrate that the optimized layout of the Alta $\mathrm{X}$ wind farm achieves a $12 \%$ increase in power output at a similar cost to the original layout.

The results obtained with the proposed approach are very promising and more appropriate to evaluate the placement of WTs in a wind farm project. In the ongoing research, we will consider more realistic wind farm conditions and more advanced wake models, such as computational fluid dynamics (CFD) models.

\section{Conflicts of interest}

The authors declare no conflict of interest.

\section{Author contributions}

Conceptualization, Mohammed Amine Hassoine; methodology, Mohammed Amine Hassoine and Abdessalam Ait Madi; software, Mohammed Amine Hassoine and Adnane Addaim; formal analysis, investigation, resources, data preparation, Mohammed Amine Hassoine; writing — original draft preparation, Mohammed Amine Hassoine; review and editing, Mohammed Amine Hassoine, Abdessalam Ait Madi, Adnane Addaim and Fouad Lahlou; supervision, Fouad Lahlou, and Adnane Addaim. 


\section{References}

[1] Our world in data, https://ourworldindata.org , [Online] [accessed: 12- April- 2021].

[2] The Covid-19 crisis is hurting but not halting global growth in renewable power capacity, https://www.iea.org/news/the-covid-19-crisisis-hurting-but-not-halting-global-growth-inrenewable-power-capacity, [Online] [accessed: 12- April- 2021].

[3] J. Lee and F. Zhao, Global wind report, Global Wind Energy Council, Brussels, Belgium, 2019, https://gwec.net/wp-

content/uploads/2020/08/Annual-Wind-

Report_2019_digital_final_2r.pdf , [Online] [accessed: 12- April- 2021].

[4] G. Mosetti, C. Poloni, and B. Diviacco, "Optimization of wind turbine positioning in large windfarms by means of a genetic algorithm", Journal of Wind Engineering and Industrial Aerodynamics, Vol. 51, No. 1, pp. 105-116, 1994.

[5] S. Grady, M. Hussaini, and M. Abdullah, "Placement of wind turbines using genetic algorithms", Renewable Energy, Vol. 30, No. 2, pp. 259-270, 2005.

[6] A. Emami and P. Noghreh, "New approach on optimization in placement of wind turbines within wind farm by genetic algorithms", Renewable Energy, Vol. 35, No. 7, pp. 15591564, 2010.

[7] C. Wan, J. Wang, G. Yang, and X. Zhang, "Optimal siting of wind turbines using realcoded genetic algorithms", In: Proc. of European Wind Energy Association Conference and Exhibition, Marseille, France, pp. 1-6, 2009.

[8] J. González, A. G. Rodriguez, J. Mora, J. Santos, and M. Payan, "Optimization of wind farm turbines layout using an evolutive algorithm", Renewable Energy, Vol. 35, No. 8, pp. 16711681, 2010.

[9] A. Mittal, Optimization of the Layout of Large Wind Farms using a Genetic Algorithm, MSc Dissertation, Case Western Reserve University, Cleveland, 2010.

[10] K. Chen, M. Song, Z. He, and X. Zhang, "Wind turbine positioning optimization of wind farm using greedy algorithm", Journal of Renewable and Sustainable Energy, Vol. 5, No. 2, pp. 023128, 2013.

[11] X. Gao, H. Yang, L. Lin, and P. Koo, "Wind turbine layout optimization using multipopulation genetic algorithm and a case study in
Hong Kong offshore", Journal of Wind Engineering and Industrial Aerodynamics, Vol. 139, pp. 89-99, 2015.

[12] M. Hassoine, F. Lahlou, A. Addaim, and A. Madi, "Wind Farm Layout Optimization using Real Coded Multi-population Genetic Algorithm", In: Proc. of International Conf. on Wireless Technologies, Embedded and Intelligent Systems, Fez, Morocco, pp. 1-5, 2019.

[13] M. Hassoine, F. Lahlou, A. Addaim, and A. Madi, "A Novel Evaluation of Wind Energy Potential in Essaouira Offshore Wind Farm, using Genetic Algorithm and MERRA-2 Reanalysis Data", In: Proc. of International Conf. on Optimization and Applications, Kenitra, Morocco, pp. 1-6, 2019.

[14] S. Pookpunt and W. Ongsakul, "Optimal placement of wind turbines within wind farm using binary particle swarm optimization with time-varying acceleration coefficients", Renewable Energy, Vol. 55, pp. 266-276, 2013.

[15] P. Asaah, L. Hao, and J. Ji, "Optimal Placement of Wind Turbines in Wind Farm Layout Using Particle Swarm Optimization", Journal of Modern Power Systems and Clean Energy, Vol. 9, No. 2, pp. 367-375, 2021.

[16] J. Bansal, and P. Farswan, "Wind farm layout using biogeography based optimization", Renewable Energy, Vol. 107, pp. 386-402, 2017.

[17] S. P. Kheljan, A. Azimi, B. M. Ivatloo, and M. Rasouli, "Optimal design of wind farm layout using a biogeographical based optimization algorithm", Journal of Cleaner Production, Vol. 201, pp. 1111-1124, 2018.

[18] S. Frandsen, "On the wind speed reduction in the center of large clusters of wind turbines", Journal of Wind Engineering and Industrial Aerodynamics, Vol. 39, No. 1-3, pp. 251-265, 1992.

[19] N. O. Jensen, A note on wind generator interaction, No. 2411, Ris $\varnothing$ National Laboratory. Risø-M. 1983.

[20] D. J. Renkema, "Validation of wind turbine wake models: Using wind farm data and wind tunnel measurements", MSc Dissertation, Delft University of Technology, Delft, 2007.

[21] Z. Shao, Y. Wu, L. Li, S. Han, and Y. Liu, "Multiple Wind Turbine Wakes Modeling Considering the Faster Wake Recovery in Overlapped Wakes", Energies, Vol. 12, No. 4, p. 680, 2019.

[22] I. Katic, J. Højstrup, and N. O. Jensen, "A Simple Model for Cluster Efficiency”, In: Proc. 
of European Wind Energy Association Conference and Exhibition, Rome, Italy, pp. 407-410, 1986.

[23] D. Simon, "Biogeography-Based Optimization", IEEE Transactions on Evolutionary Computation, Vol. 12, No. 6, pp. 702-713, 2008.

[24] P. Yin, T. Wu, and P. Hsu, "Risk management of wind farm micro-siting using an enhanced genetic algorithm with simulation optimization", Renewable Energy, Vol. 107, pp. 508-521, 2017.

[25] Top 10 biggest wind farms, https://www.powertechnology.com/features/feature-biggest-windfarms-in-the-world-texas, [Online] [accessed: 12- April- 2021].

[26] P. Riley, GCEP Energy tutorial wind 101, Stanford University, Stanford, USA, 2014, https://gwec.net/wpcontent/uploads/2020/08/Annual-WindReport_2019_digital_final_2r.pdf, [Online] [accessed: 02- May- 2021].

[27] GE General Electric GE 2.85 - 103, https://en.wind-turbinemodels.com/turbines/750-ge-general-electricge-2.85-103, [Online] [accessed: 02- May2021].

[28] D. Deaves and I. Lines, "On the fitting of low mean windspeed data to the Weibull distribution", Journal of Wind Engineering and Industrial Aerodynamics, Vol. 66, No. 3, pp. 169-178, 1997.

[29] D. Kang, K. Ko, and J. Huh, "Comparative Study of Different Methods for Estimating Weibull Parameters: A Case Study on Jeju Island, South Korea", Energies, Vol. 11, No. 2, p. 356, 2018.

[30] The Wind Prospector, https://maps.nrel.gov/wind-prospector, [Online] [accessed: 02- May- 2021].

[31] Electricity data browser, https://www.eia.gov/electricity/data/browser/ , [Online] [accessed: 02- May- 2021]. 